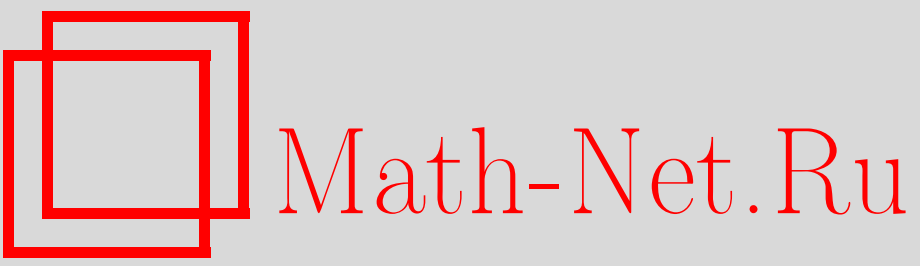

А. С. Парван, Алгебраический метод точного решения статистической суммы для канонического ансамбля в ядерной мультифрагментации, ТM $\Phi, 2004$, том 140, номер 1, 100-112

DOI: https://doi.org/10.4213/tmf81

Использование Общероссийского математического портала Math-Net.Ru подразумевает, что вы прочитали и согласны с пользовательским соглашением

http://www . mathnet.ru/rus/agreement

Параметры загрузки:

IP : 3.89 .197 .203

26 апреля 2023 г., 18:28:59 
ТЕОРЕТИЧЕСКАЯ

И МАТЕМАТИЧЕСКАЯ

ФИЗИКА

Том 140, № 1

июль, 2004

(C) 2004 г.

А. С. Парван*†

\title{
АЛГЕБРАИЧЕСКИЙ МЕТОД ТОЧНОГО РЕШЕНИЯ СТАТИСТИЧЕСКОЙ СУММЫ ДЛЯ КАНОНИЧЕСКОГО АНСАМБЛЯ В ЯДЕРНОЙ МУЛЬТИФРАГМЕНТАЦИИ
}

\begin{abstract}
Предложен единый метод вывода рекуррентных уравнений для канонической статистической суммы системы невзаимодействующих частиц с сохраняющимися зарядами в случае, ког да частицы подчиняются статистике Бозе-Эйнштейна, Ферми-Дирака, Максвелла-Больцмана или парастатистике. Для всех этих типов статистик получены рекуррентные соотношения для статистической суммы новой статистической модели ядерной мультифрагментации с сохраняющимися барионным и электрическим зарядами при учете внутренних степеней свободы ядерных фрагментов.
\end{abstract}

Ключевые слова: каноническая статистическая сумма, квантовая статистика, рекуррентные соотношения, ядерная мультифрагментация.

\section{1. ВВЕДЕНИЕ}

Методы равновесной статистической механики широко применяются в физике столкновений тяжелых ионов при промежуточных и высоких энергиях для анализа выхода частищ и исследования термодинамических свойств (уравнений состояния) конечных ядерных систем. Точный учет сохраняюшихся зарядов важен для описания явлений ядерной мультифрагментации [1], [2] и процессов выхода частиц в ультрарелятивистских столкновениях тяжелых ионов [3], [4]. В последнее время применение этих законов сохранения в рамках статистических моделей стало возможным благодаря развитию методов точного вычисления статистической суммы канонического ансамбля. Точные решения для статистической суммы канонического ансамбля на основе рекуррентных уравнений [5] известны для очень узкого класса задач, таких как квантовый идеальный газ $N$ тождественных частиц [6]-[9] и статистические модели мультифрагментации с классической статистикой [10]-[12]. В работах [10], [11], [13] рассматривались рекуррентные уравнения в приложении к ядерной мультифрагментации для системы нукло-

\footnotetext{
* Объединенный институт ядерных исследований, Дубна, Московская обл., Россия. E-mail: parvan@thsun1.jinr.ru

${ }^{\dagger}$ Институт прикладной физики Академии наук Республики Молдова, Кишинев, Молдова
} 
нов с сохраняющимся барионным зарядом. Для системы нейтронов и протонов с сохраняюшимися барионным и электрическим зарядами рекуррентные уравнения были выведены в работе [12] и применены к изучению мультифрагментации в работах [14], [15]. Для статистической модели мультифрагментации с квантовой статистикой точные решения известны только в случае сохранения барионного заряда [9].

В данной работе найден новый алгебраический метод вывода рекуррентных уравнений для статистической суммы канонического ансамбля в представлении чисел заполнения в случаях квантовой, классической статистики и парастатистики. На основе этого подхода удается получить рекуррентные соотношения для статистической суммы и средних по ансамблю в рамках новой статистической модели ядерной мультифрагментации с сохранением барионного и электрического зарядов для статистик Бозе-Эйнштейна, Ферми-Дирака и парастатистики при учете внутренних степеней свободы ядерных фрагментов. Следует отметить преимущества этой модели по сравнению с другими статистическими моделями ядерной мультифрагментации: а) в используемом каноническом ансамбле точно учтено сохранение барионного и электрического зарядов; б) модель обобщается на случай квантовой статистики и парастатистики фрагментов; в) развитая модель допускает точное решение задачи. Статистическая сумма этой модели приводится к обобшенной сумме с мультиномиальными коэффициентами. Отметим также, что как для модели мультифрагментации с сохранением барионного заряда, так и для идеального газа $N$ тождественных частиц статистическая сумма приводится к сумме с мультиномиальными коэффициентами [5] независимо от статистики фрагментов (частиц).

В разделе 2 рассмотрен идеальньй газ $N$ тождественных частиц в каноническом ансамбле и сформулирован метод вывода рекуррентных соотношений. В разделе 3 этот метод обобщается на случай статистической модели мультифрагментации с сохранением барионного заряда. В разделе 4 сформулирована статистическая модель мультифрагментации с сохранением барионного и электрического зарядов, для статистической суммы которой приведены соответствуюшие рекуррентные соотношения. В разделе 5 содержится обсуждение полученных результатов и сделаны некоторые заключительные замечания. В приложениях дается вывод рекуррентных уравнений и доказательство основного тождества.

\section{2. ИДЕАЛЬНЫЙ ГАЗ $N$ ТОЖДЕСТВЕННЫХ ЧАСТИЦ. КАНОНИЧЕСКИЙ АНСАМБЛЬ}

Статистическая сумма для идеального газа $N$ тождественных частиц в каноническом ансамбле дается формулой [16]

$$
\mathcal{Q}_{N}=\sum_{\left\{n_{\vec{p}}\right\}} \delta\left(\sum_{\vec{p}} n_{\vec{p}}-N\right) G\left(\left\{n_{\vec{p}}\right\}\right) e^{-\beta \sum_{\vec{p}} \varepsilon_{\vec{p}} n_{\vec{p}}},
$$

где $\beta=1 / T, \varepsilon_{\vec{p}}-$ одночастичные энергии, $\delta$ - символ Кронекера. Допустимы следующие значения $n_{\vec{p}}$ :

$$
n_{\vec{p}}=0,1,2, \ldots, K
$$


где $K=1$ для статистики Ферми-Дирака, $K=N$ для статистик Бозе-Эйнштейна и Максвелла-Больцмана. Число состояний системы, соответствуюших $\left\{n_{\vec{p}}\right\}$, есть $G\left(\left\{n_{\vec{p}}\right\}\right)=1$ для статистик Ферми-Дирака и Бозе-Эйнштейна и $G\left(\left\{n_{\vec{p}}\right\}\right)=1 / \prod_{\vec{p}} n_{\vec{p}}$ ! для статистики Максвелла-Больцмана с "правильным больцмановским подсчетом". Отметим, что для конкретной физической системы задается соответствующий набор квантовых чисел и энергий одночастичных состояний. Кроме того, в данной работе рассматривается исключительно математическая задача, когда частицы подчиняются парастатистике [17], для которой $1<K<N$ и $G\left(\left\{n_{\vec{p}}\right\}\right)=1$.

Статистическая сумма (1) удовлетворяет следуюшим рекуррентным соотношениям [6]-[9]:

$$
\begin{aligned}
\mathcal{Q}_{N} & =\frac{1}{N} \sum_{l=1}^{N} \omega_{l} \mathcal{Q}_{N-l}, \\
\omega_{l} & =y_{l} \sum_{\vec{p}} e^{-\beta l \varepsilon_{\vec{p}}}
\end{aligned}
$$

где $\mathcal{Q}_{0}=1, y_{l}=(\mp 1)^{l+1}$ (здесь и в дальнейшем верхний знак соответствует статистике Ферми-Дирака, а нижний - статистике Бозе-Эйнштейна), $y_{l}=\delta_{l, 1}$ для статистики Максвелла-Больцмана. Заметим, что для парастатистики $y_{l}=1-(K+1) \delta(l /(K+1)-$ $[l /(K+1)])$. Здесь и далее $[a]$ означает целую часть числа $a$.

Докажем справедливость рекуррентных уравнений (3) для статистической суммы (1). Можно доказать следуюшее тождество (см. приложение А):

$$
\sum_{n_{\vec{p}}=0}^{K} n_{\vec{p}} G\left(n_{\vec{p}}\right) x^{n_{\vec{p}}} \Phi\left(N-n_{\vec{p}}\right)=\sum_{l=1}^{N} y_{l} x^{l} \sum_{n_{\vec{p}}=0}^{\min [N-l, K]} G\left(n_{\vec{p}}\right) x^{n_{\vec{p}}} \Phi\left(N-n_{\vec{p}}-l\right),
$$

где $x, \Phi$ - произвольные переменные. В силу закона сохранения полного числа частиц $\sum_{\vec{p}} n_{\vec{p}}=N$ статистическая сумма (1) принимает следуюший вид

$$
\mathcal{Q}_{N}=\frac{1}{N} \sum_{\vec{p}^{\prime}} \sum_{\left\{n_{\vec{p}}\right\}} \delta\left(\sum_{\vec{p}} n_{\vec{p}}-N\right) n_{\vec{p}^{\prime}} G\left(\left\{n_{\vec{p}}\right\}\right) e^{-\beta \sum_{\vec{p}} \varepsilon_{\vec{p}} n_{\vec{p}}}
$$

Заметим, что сумма по $n_{\vec{p}^{\prime}}$ в выражении (6) совпадает с левой частью тождества (5), если в (5) положить $x=e^{-\beta \varepsilon_{\vec{p}^{\prime}}}$ и $\Phi\left(N-n_{\vec{p}^{\prime}}\right)=\delta\left(\sum_{\vec{p} \neq \vec{p}^{\prime}} n_{\vec{p}}-\left(N-n_{\vec{p}^{\prime}}\right)\right)$,

$$
\begin{aligned}
\mathcal{Q}_{N}= & \frac{1}{N} \sum_{\vec{p}^{\prime}} \sum_{\left\{n_{\vec{p}}\right\}_{\vec{p} \neq \vec{p}^{\prime}}} G\left(\left\{n_{\vec{p}}\right\}_{\vec{p} \neq \vec{p}^{\prime}}\right) e^{-\beta \sum_{\vec{p} \neq \vec{p}^{\prime}} \varepsilon_{\vec{p}} n_{\vec{p}} \times} \\
& \times \sum_{n_{\vec{p}^{\prime}}=0}^{K} n_{\vec{p}^{\prime}} G\left(n_{\vec{p}^{\prime}}\right) e^{-\beta \varepsilon_{\vec{p}^{\prime}} n_{\vec{p}^{\prime}} \delta\left(\sum_{\vec{p} \neq \vec{p}^{\prime}} n_{\vec{p}}-\left(N-n_{\vec{p}^{\prime}}\right)\right) .} .
\end{aligned}
$$


Используя тождество (5) и уравнения (1), (4), окончательно получаем рекуррентные уравнения (3),

$$
\begin{aligned}
& \mathcal{Q}_{N}=\frac{1}{N} \sum_{\vec{p}^{\prime}} \sum_{\left\{n_{\vec{p}}\right\}_{\vec{p} \neq \vec{p}^{\prime}}} G\left(\left\{n_{\vec{p}}\right\}_{\vec{p} \neq \vec{p}^{\prime}}\right) e^{-\beta \sum_{\vec{p} \neq \vec{p}^{\prime}} \varepsilon_{\vec{p}} n_{\vec{p}}} \sum_{l=1}^{N} y_{l} e^{-\beta l \varepsilon_{\vec{p}^{\prime}} \times} \\
& \times \sum_{n_{\vec{p}^{\prime}}=0}^{\min [N-l, K]} G\left(n_{\vec{p}^{\prime}}\right) e^{-\beta \varepsilon_{\vec{p}^{\prime}} n_{\vec{p}^{\prime}} \delta}\left(\sum_{\vec{p} \neq \vec{p}^{\prime}} n_{\vec{p}}-\left(N-n_{\vec{p}^{\prime}}-l\right)\right)= \\
& =\frac{1}{N} \sum_{l=1}^{N} y_{l} \sum_{\vec{p}^{\prime}} e^{-\beta l \varepsilon_{\vec{p}^{\prime}}} \sum_{\left\{n_{\vec{p}}\right\}} \delta\left(\sum_{\vec{p}} n_{\vec{p}}-(N-l)\right) G\left(\left\{n_{\vec{p}}\right\}\right) e^{-\beta \sum_{\vec{p}} \varepsilon_{\vec{p}} n_{\vec{p}}}= \\
& =\frac{1}{N} \sum_{l=1}^{N} \omega_{l} \mathcal{Q}_{N-l}
\end{aligned}
$$

Воспользовавшись тем же приемом, что и при выводе формулы (8), можно получить следующие представления для средних:

$$
\begin{aligned}
\left\langle n_{\vec{p}}\right\rangle_{N}= & \frac{1}{\mathcal{Q}_{N}} \sum_{\left\{n_{\vec{p}}\right\}} \delta\left(\sum_{\vec{p}} n_{\vec{p}}-N\right) n_{\vec{p}} G\left(\left\{n_{\vec{p}}\right\}\right) e^{-\beta \sum_{\vec{p}} \varepsilon_{\vec{p}} n_{\vec{p}}}= \\
= & \frac{1}{\mathcal{Q}_{N}} \sum_{k=1}^{N} y_{k} e^{-\beta k \varepsilon_{\vec{p}} \mathcal{Q}_{N-k},} \\
\left\langle n_{\vec{p}} n_{\vec{p}^{\prime}}\right\rangle_{N}= & \frac{1}{\mathcal{Q}_{N}} \sum_{\left\{n_{\vec{p}}\right\}} \delta\left(\sum_{\vec{p}} n_{\vec{p}}-N\right) n_{\vec{p}} n_{\vec{p}^{\prime}} G\left(\left\{n_{\vec{p}}\right\}\right) e^{-\beta \sum_{\vec{p}} \varepsilon_{\vec{p}} n_{\vec{p}}}= \\
= & \frac{1}{\mathcal{Q}_{N}} \sum_{i=1}^{N} \sum_{j=1}^{N-i} y_{i} y_{j} e^{-\beta i \varepsilon_{\vec{p}}} e^{-\beta j \varepsilon_{\vec{p}^{\prime}} \mathcal{Q}_{N-i-j}+} \\
& +\delta_{\vec{p} \vec{p}^{\prime}} \frac{1}{\mathcal{Q}_{N}} \sum_{j=1}^{N} y_{j} e^{-\beta j \varepsilon_{\vec{p}} j \mathcal{Q}_{N-j}}
\end{aligned}
$$

Путем последовательного использования рекуррентного уравнения (3) статистическую сумму $\mathcal{Q}_{N}$ можно представить в виде суммы с мультиномиальными коэффициентами [5], [16]

$$
\mathcal{Q}_{N}=\sum_{\left\{n_{k}\right\}} \delta\left(\sum_{k=1}^{N} k n_{k}-N\right) \prod_{k=1}^{N} \frac{\omega_{k}^{n_{k}}}{k^{n_{k} n_{k} !}} .
$$

Сумма по $\left\{n_{k}\right\}$ в (11) берется по всем наборам неотрицательных целых чисел от $n_{1}$ до $n_{N}$ таким, что $N=n_{1}+2 n_{2}+\cdots+N n_{N}$, или, что то же самое, по всем разбиениям числа $N$, или по всем цикловым классам $\left(n_{1}, \ldots, n_{N}\right)$ перестановок $N$ элементов. В комбинаторном анализе мультиномиальный коэффишиент

$$
C\left(N,\left\{n_{k}\right\}\right)=\frac{N !}{\prod_{k=1}^{N} k^{n_{k}} n_{k} !}
$$


представляет собой число перестановок из $N$ элементов класса $\left(n_{1}, \ldots, n_{N}\right)$. Перестановка $N$ символов описывается симметрической группой $S_{N}$, которая имеет $N$ ! элементов. При представлении перестановок в виде циклов число цикловых классов равно числу неприводимых представлений группы $S_{N}[18],[19]$. Производящая функция $N ! \mathcal{Q}_{N}\left(\omega_{1}, \ldots, \omega_{N}\right)$ чисел $C\left(N,\left\{n_{k}\right\}\right)$ носит название циклового индикатора симметрической группы $S_{N}[5]$.

Определим статистическую сумму для большого канонического ансамбля. С учетом (11) большую статистическую сумму можно записать в виде

$$
\mathcal{Q}^{\mathrm{GC}}(\lambda) \equiv \sum_{N=0}^{\infty} \lambda^{N} \mathcal{Q}_{N}=e^{\sum_{k=1}^{\infty} k^{-1} \omega_{k} \lambda^{k}}
$$

где $\lambda=e^{\beta \mu}, \mu$ - химический потенциал.

Отметим, что статистическая сумма $\mathcal{Q}_{N}$, выраженная в терминах $x_{\vec{p}}=e^{-\beta \varepsilon_{\vec{p}}}$, в случае статистики Бозе-Эйнштейна является полностью симметрической функцией, а в случае статистики Ферми-Дирака - элементарной симметрической функцией [20], [21].

\section{3. СТАТИСТИЧЕСКАЯ МОДЕЛЬ ЯДЕРНОЙ МУЛЬТИФРАГМЕНТАЦИИ С ТОЧНЫМ СОХРАНЕНИЕМ БАРИОННОГО ЗАРЯДА СИСТЕМЫ}

Для модели ядерной мультифрагментации с законом сохранения барионного заряда в случае, когда фрагменты подчиняются квантовой или классической статистике, статистическая сумма системы и ее рекуррентные уравнения представляются в следуюшем виде [9]:

$$
\begin{aligned}
\mathcal{Q}_{A} & =\sum_{\left\{N_{k \vec{p}}\right\}} \delta\left(\sum_{k=1}^{A} \sum_{\vec{p}} k N_{k \vec{p}}-A\right) G\left(\left\{N_{k \vec{p}}\right\}\right) e^{-\beta \sum_{k \vec{p}} E_{k \vec{p}} N_{k \vec{p}}}= \\
& =\frac{1}{A} \sum_{k=1}^{A} \sum_{\mu=1}^{[A / k]} k \omega_{k \mu} \mathcal{Q}_{A-k \mu}= \\
& =\frac{1}{A} \sum_{k=1}^{A} \omega_{k} \mathcal{Q}_{A-k},
\end{aligned}
$$

где $\mathcal{Q}_{0}=1,[A / k]$ - максимально возможное число фрагментов сорта $k$ для системы из $A$ нуклонов и $E_{k \vec{p}}-$ энергия фрагмента с барионным зарядом $k$. Допустимы следуюшие значения для чисел заполнения $N_{k \vec{p}}$ :

$$
N_{k \vec{p}}=0,1,2, \ldots, K_{k},
$$

где целое число $K_{k}=1$ для статистики Ферми-Дирака, $K_{k}=[A / k]$ для статистик Бозе-Эйнштейна и Максвелла-Больцмана. Отметим, что в случае парастатистики 
$1<K_{k}<[A / k]$. Число состояний системы $G\left(\left\{N_{k \vec{p}}\right\}\right)$ определяется по формулам из предыдушего раздела в виде произведения по всем сортам фрагментов. Переменные $\omega_{k \mu}$ и $\omega_{k}$ записьваются как

$$
\begin{aligned}
\omega_{k \mu} & =y_{k \mu} \sum_{\vec{p}} e^{-\beta \mu E_{k \vec{p}}} \\
\omega_{k} & =\sum_{l=1}^{k} \sum_{\mu=1}^{k} \delta_{k, l \mu} l y_{l \mu} \sum_{\vec{p}} e^{-\beta \mu E_{l \vec{p}}}=\sum_{l=1}^{k} \sum_{\mu=1}^{k} \delta_{k, l \mu} l \omega_{l \mu},
\end{aligned}
$$

где величины $y_{k \mu}$ совпадают с переменными $y_{\mu}$, введенными в предыдущем разделе, для каждой из подсистем тождественных фрагментов сорта $k$ с заменой $K$ на $K_{k}$.

Вывод рекуррентного уравнения (15) для статистической суммы (14) приведен в приложении Б и основан на использовании тождества

$$
\begin{aligned}
& \sum_{N_{k \vec{p}}=0}^{K_{k}} N_{k \vec{p}} G\left(N_{k \vec{p}}\right) x^{N_{k \vec{p}}} \Phi\left(A-k N_{k \vec{p}}\right)= \\
& \quad=\sum_{\mu=1}^{[A / k]} y_{k \mu} x^{\mu} \sum_{N_{k \vec{p}}=0}^{\min \left([A / k]-\mu, K_{k}\right)} G\left(N_{k \vec{p}}\right) x^{N_{k \vec{p}}} \Phi\left(A-k N_{k \vec{p}}-k \mu\right),
\end{aligned}
$$

справедливость которого можно проверить непосредственно, как и для случая тождества (5). На основе тождества (20) и алгебраического метода суммирования статистической суммы $\mathcal{Q}_{A}$ (см. приложение Б) можно вычислить любое среднее по ансамблю.

Путем последовательного использования рекуррентного уравнения (16) статистическую сумму $\mathcal{Q}_{A}$ можно записать в виде суммы (ср. с (11))

$$
\mathcal{Q}_{A}=\sum_{\left\{N_{k}\right\}} \delta\left(\sum_{k=1}^{A} k N_{k}-A\right) \prod_{k=1}^{A} \frac{\omega_{k}^{N_{k}}}{k^{N_{k} N_{k} !}} .
$$

Статистическая сумма $A ! \mathcal{Q}_{A}\left(\omega_{1}, \ldots, \omega_{A}\right)$ является цикловым индикатором симметрической группы $S_{A}$ или производяшей функцией мультиномиальных коэффициентов $C\left(A,\left\{N_{k}\right\}\right)(12)$. Отметим, что в предельном случае $y_{k \mu} \rightarrow \delta_{k, 1} y_{k \mu}$ все проинтегрированные выражения статистической модели ядерной мультифрагментации принимают вид, соответствуюший идеальному газу $A$ тождественных частиц.

Большая статистическая сумма $\mathcal{Q}^{\mathrm{GC}}(\lambda)$ выражается в форме $(13)$ с функциями $\omega_{k}$ из (18) и (19). Используя интегральное представление для символа Кронекера

$$
\frac{1}{2 \pi} \int_{0}^{2 \pi} d \phi e^{i \phi\left(\sum_{k} k N_{k}-A\right)}=\delta\left(\sum_{k} k N_{k}-A\right),
$$

статистическую сумму (21) можно представить в виде

$$
\begin{aligned}
\mathcal{Q}_{A} & =\frac{1}{2 \pi} \int_{0}^{2 \pi} d \phi e^{-i \phi A} \widetilde{\mathcal{Q}}(\phi), \\
\widetilde{\mathcal{Q}}(\phi) & \equiv \mathcal{Q}^{\mathrm{GC}}\left(\lambda \rightarrow e^{i \phi}\right)=e^{\sum_{k=1}^{\infty} k^{-1} \omega_{k} e^{i \phi k}},
\end{aligned}
$$


где $\phi \in[0,2 \pi]$ - параметр группы $U(1)$. Такой метод вычисления статистической суммы $\mathcal{Q}_{A}$ является частным случаем более обшего группового метода, применяюшегося к системам с любой внутренней симметрией, представленной полупростой алгеброй Ли [22], [23]. Отметим, что сохранение $N$ числа частиц для идеального газа соответствует $U(1)$-симметрии [20].

\section{4. СТАТИСТИЧЕСКАЯ МОДЕЛЬ ЯДЕРНОЙ МУЛЬТИФРАГМЕНТАЦИИ С ТОЧНЫМ СОХРАНЕНИЕМ БАРИОННОГО И ЭЛЕКТРИЧЕСКОГО ЗАРЯДОВ СИСТЕМЫ}

В рамках равновесной статистической механики в каноническом ансамбле сформулируем статистическую модель ядерной мультифрагментации с точным учетом законов сохранения барионного и электрического зарядов с квантовой статистикой фрагментов [24]. Пусть задана ядерная система $(A, Z)$, размешенная в объеме $V$ при температуpe $T$. Сорт фрагмента (частицы) задается двумя целыми числами $(k, l)$, где $k$ - число нуклонов, $l$ - число протонов, $k-l$ - число нейтронов. Для ядерной системы $(A, Z)$ числа $k, l$ фиксируют определенньй сорт фрагмента и принимают значения $l=0,1, \ldots, Z$; $k=l, l+1, \ldots, A-Z+l, k \neq 0$. Полное число разрешенных сортов фрагментов системы $(A, Z)$

$$
\mathcal{N}_{A Z}=(A-Z+1)(Z+1)-1=(A-Z) Z+A
$$

Состояние фрагмента сорта $(k, l)$ фиксируется набором квантовых чисел $\mathbf{a}=\left(\xi, \vec{p}, I_{z}\right)$, где $\xi$ - номер уровня внутреннего возбуждения этого фрагмента, $\vec{p}-$ его импульс и $I_{z}-$ проекция спина на ось $z$ для фрагмента сорта $(k, l)$ на уровне внутреннего возбуждения $\xi$. Квантовые числа из набора а принимают следующие значения:

$$
\begin{aligned}
\xi & =0,1,2, \ldots, \\
p^{(\alpha)} & =\Delta p^{(\alpha)} n^{(\alpha)}, \quad n^{(\alpha)}=0, \pm 1, \ldots, \quad \alpha=x, y, z, \quad \Delta p^{(\alpha)}=\frac{2 \pi \hbar}{L} \\
I_{z} & =-S_{k l \xi}, \ldots, S_{k l \xi}
\end{aligned}
$$

где $\xi=0$ соответствует уровню основного состояния фрагмента сорта $(k, l), \xi=1$ - первому возбужденному уровню и.т.д., $L=V^{1 / 3}, S_{k l \xi}$ - спин фрагмента сорта $(k, l)$ с внутренним возбуждением на уровне $\xi$.

Микросостояние системы задается набором чисел заполнения $\left\{N_{k l \mathbf{a}}\right\}$ одночастичных состояний фрагментов $\{k l \mathbf{a}\}$. Число $N_{k l \mathbf{a}}$ представляет собой число фрагментов сорта $(k, l)$ в состоянии а. Энергия микросостояния системы

$$
E=\sum_{k l \mathbf{a}} N_{k l \mathbf{a}} E_{k l \mathbf{a}}
$$

где энергия фрагмента

$$
E_{k l \mathbf{a}}=\frac{\vec{p}^{2}}{2 m_{k l}}+B_{k l}+E_{k l \xi}^{*}+\Delta E_{k l}
$$


$m_{k l}=(k-l) m_{n}+l m_{p} \simeq k m-$ масса фрагмента из $k-l$ нейтронов массы $m_{n}$ и $l$ протонов массы $m_{p} ; m$ - масса нуклона; $B_{k l}$ - энергия связи фрагмента сорта $(k, l) ; E_{k l \xi}^{*}$ - энергия его внутреннего возбуждения на уровне $\xi ; \Delta E_{k l}$ - энергия взаимодействия фрагмента со средним полем.

Набор чисел заполнения $\left\{N_{k l}\right\}$ должен удовлетворять законам сохранения барионного $A$ и электрического $Z$ зарядов ядерной системы,

$$
\begin{aligned}
& \sum_{l=0}^{Z} \sum_{k=l, k \neq 0}^{A-Z+l} \sum_{\mathbf{a}} k N_{k l \mathbf{a}}=A, \\
& \sum_{l=0}^{Z} \sum_{k=l, k \neq 0}^{A-Z+l} \sum_{\mathbf{a}} l N_{k l \mathbf{a}}=Z .
\end{aligned}
$$

Максимально возможное число фрагментов $\widetilde{N}_{k l}$ сорта $(k, l)$ в системе $(A, Z)$ вычисляется по формуле

$$
\widetilde{N}_{k l}= \begin{cases}{\left[\frac{A-Z}{k-l}\right],} & l=0 \\ {\left[\frac{Z}{l}\right],} & k=l \neq 0 \\ \min \left(\left[\frac{Z}{l}\right],\left[\frac{A-Z}{k-l}\right]\right), & k \neq l \neq 0\end{cases}
$$

Числа заполнения для каждого сорта $(k, l)$ принимают значения

$$
N_{k l \mathbf{a}}=0,1, \ldots, K_{k l},
$$

где $K_{k l}=1$ для статистики Ферми-Дирака, $K_{k l}=\widetilde{N}_{k l}$ для статистик Бозе-Эйнштейна и Максвелла-Больцмана.

Статистическая сумма модели в представлении чисел заполнения имеет вид

$$
\mathcal{Q}_{A, Z}=\sum_{\left\{N_{k l \mathbf{a}}\right\}} \delta\left(\sum_{k l \mathbf{a}} k N_{k l \mathbf{a}}-A\right) \delta\left(\sum_{k l \mathbf{a}} l N_{k l \mathbf{a}}-Z\right) G\left(\left\{N_{k l \mathbf{a}}\right\}\right) e^{-\beta \sum_{k l \mathbf{a}} E_{k l \mathbf{a}} N_{k l \mathbf{a}}}
$$

где число состояний системы $G\left(\left\{N_{k l \mathbf{a}}\right\}\right)=1$ для квантовой статистики и $G\left(\left\{N_{k l \mathbf{a}}\right\}\right)=$ $(1 / A !) A ! / \prod_{k l \mathbf{a}} N_{k l \mathbf{a}}$ ! для классической статистики с "правильным больцмановским подсчетом". Заметим, что для парастатистики $1<K_{k l}<\widetilde{N}_{k l}$ и $G\left(\left\{N_{k l \mathbf{a}}\right\}\right)=1$.

Нетрудно показать, что для статистической суммы (32) выполняются следующие рекуррентные уравнения:

$$
\begin{aligned}
\mathcal{Q}_{A, Z} & =\frac{1}{A} \sum_{l=0}^{Z} \sum_{k=l, k \neq 0}^{A-Z+l} \sum_{\mu=1}^{\tilde{N}_{k l}} k \omega_{k l \mu} \mathcal{Q}_{A-k \mu, Z-l \mu}= \\
& =\frac{1}{A} \sum_{l=0}^{Z} \sum_{k=l, k \neq 0}^{A-Z+l} k \omega_{k l} \mathcal{Q}_{A-k, Z-l},
\end{aligned}
$$


где $\mathcal{Q}_{0,0}=1$. Величины $\omega_{k l \mu}$ и $\omega_{k l}$ выражаются через энергии фрагментов $(27)$ в форме

$$
\begin{aligned}
\omega_{k l} & =\sum_{i=1}^{k} \sum_{j=0}^{l} \sum_{\mu=1}^{k} \delta_{k, i \mu} \delta_{l, j \mu}\left(\frac{i}{k}\right) \sum_{\mathbf{a}} y_{i j \mathbf{a} \mu} e^{-\beta \mu E_{i j \mathbf{a}}}, \\
\omega_{k l \mu} & =\sum_{\mathbf{a}} y_{k l \mathbf{a} \mu} e^{-\beta \mu E_{k l \mathbf{a}}},
\end{aligned}
$$

где $y_{k l \mathbf{a} \mu}=(\mp 1)^{\mu+1}$ для квантовой статистики и $y_{k l \mathbf{a} \mu}=\delta_{\mu, 1}$ для классической статистики фрагментов сорта $(k, l)$ на уровне внутреннего возбуждения $\xi$. В случае, когда фрагменты подчиняются парастатистике, $y_{k l \mathbf{a} \mu}=1-\left(K_{k l}+1\right) \delta\left(\mu /\left(K_{k l}+1\right)-\left[\mu /\left(K_{k l}+\right.\right.\right.$ $1)]$ ). Следует отметить, что рекуррентные соотношения (33) и (34) выполняются независимо от явного вида энергий фрагментов (27).

Рекуррентные уравнения (34) для статистической суммы $\mathcal{Q}_{A, Z}$ были получены описанным выше методом (см. также приложение Б) на основе следующего тождества, справедливость которого нетрудно проверить простой подстановкой:

$$
\begin{aligned}
\sum_{N_{k l \mathbf{a}}=}^{K_{k l}} N_{k l \mathbf{a}} G\left(N_{k l \mathbf{a}}\right) x^{N_{k l \mathbf{a}}} \Phi_{1}\left(A-k N_{k l \mathbf{a}}\right) \Phi_{2}\left(Z-l N_{k l \mathbf{a}}\right)= \\
=\sum_{\mu=1}^{\tilde{N}_{k l}} y_{k l \mathbf{a} \mu} x^{\mu} \sum_{N_{k l \mathbf{a}}=0}^{\min \left(\tilde{N}_{k l}-\mu, K_{k l}\right)} G\left(N_{k l \mathbf{a}}\right) x^{N_{k l} \times \mathbf{a} \times} \\
\quad \times \Phi_{1}\left(A-k N_{k l \mathbf{a}}-k \mu\right) \Phi_{2}\left(Z-l N_{k l \mathbf{a}}-l \mu\right),
\end{aligned}
$$

где $x, \Phi_{1}, \Phi_{2}$ - произвольные переменные.

С помошью рекуррентного уравнения (34) для статистической суммы системы получаем следуюшее представление:

$$
\mathcal{Q}_{A, Z}=\sum_{\left\{N_{k l}\right\}} \delta\left(\sum_{k l} k N_{k l}-A\right) \delta\left(\sum_{k l} l N_{k l}-Z\right) \prod_{k l} \frac{\omega_{k l}^{N_{k l}}}{N_{k l} !}
$$

Среднее по каноническому ансамблю для идеального газа ядерных фрагментов выражается обычным образом:

$$
\begin{aligned}
\langle\mathcal{O}\rangle_{A, Z}= & \frac{1}{\mathcal{Q}_{A, Z}} \sum_{\left\{N_{k l \mathbf{a}}\right\}} \delta\left(\sum_{k l \mathbf{a}} k N_{k l \mathbf{a}}-A\right) \delta\left(\sum_{k l \mathbf{a}} l N_{k l \mathbf{a}}-Z\right) \times \\
& \times \mathcal{O}\left\{N_{k l \mathbf{a}}\right\} G\left(\left\{N_{k l \mathbf{a}}\right\}\right) e^{-\beta \sum_{k l \mathbf{a}} E_{k l \mathbf{a}} N_{k l \mathbf{a}}}
\end{aligned}
$$


Приведем выражения для некоторых наиболее важных средних по ансамблю, следуюшие из формулы (39):

$$
\begin{aligned}
\left\langle N_{k l \mathbf{a}}\right\rangle_{A, Z}= & \frac{1}{\mathcal{Q}_{A, Z}} \sum_{\mu=1}^{\tilde{N}_{k l}} y_{k l \mathbf{a} \mu} e^{-\beta \mu E_{k l \mathbf{a}}} \mathcal{Q}_{A-k \mu, Z-l \mu} \\
\left\langle N_{k l \mathbf{a}} N_{i j \mathbf{b}}\right\rangle_{A, Z}= & \frac{1}{\mathcal{Q}_{A, Z}} \sum_{\mu, \nu} y_{k l \mathbf{a} \mu} y_{i j \mathbf{b} \nu} e^{-\beta \mu E_{k l \mathbf{a}}} e^{-\beta \nu E_{i j \mathbf{b}} \mathcal{Q}_{A-k \mu-i \nu, Z-l \mu-j \nu}+} \\
& +\delta_{k, i} \delta_{l, j} \delta_{\mathbf{a}, \mathbf{b}} \frac{1}{\mathcal{Q}_{A, Z}} \sum_{\mu=1}^{\tilde{N}_{k l}} \mu y_{k l \mathbf{a} \mu} e^{-\beta \mu E_{k l \mathbf{a}}} \mathcal{Q}_{A-k \mu, Z-l \mu} \\
\left\langle N_{k l}\right\rangle_{A, Z}= & \frac{1}{\mathcal{Q}_{A, Z}} \sum_{\mu=1}^{\tilde{N}_{k l}} \omega_{k l \mu} \mathcal{Q}_{A-k \mu, Z-l \mu} \\
\left\langle N_{k l} N_{i j}\right\rangle_{A, Z}= & \frac{1}{\mathcal{Q}_{A, Z}} \sum_{\mu, \nu} \omega_{k l \mu} \omega_{i j \nu} \mathcal{Q}_{A-k \mu-i \nu, Z-l \mu-j \nu}+ \\
& +\delta_{k, i} \delta_{l, j} \frac{1}{\mathcal{Q}_{A, Z}} \sum_{\mu=1}^{\tilde{N}_{k l}} \mu \omega_{k l \mu} \mathcal{Q}_{A-k \mu, Z-l \mu}
\end{aligned}
$$

Конкретные вычисления статистической суммы $\mathcal{Q}_{A, Z}$ и средних по ансамблю производятся путем интегрирования выражений (35) и (36) с определенным видом энергий фрагментов (27) [9], [14], [24].

\section{5. ЗАКЛЮЧЕНИЕ}

В данной работе на примере идеального газа $N$ тождественных частиц в каноническом ансамбле сформулирован новый метод вывода рекуррентных уравнений для статистической суммы системы в случае статистик Бозе-Эйнштейна, Ферми-Дирака, Максвелла-Больцмана и парастатистики в представлении чисел заполнения. Показано, что статистическая сумма системы для всех четырех типов статистики приводится к хорошо известной в комбинаторном анализе сумме с мультиномиальными коэффициентами [5], в которой переменные $y_{l}=(\mp 1)^{l+1}$ для статистик Ферми-Дирака и Бозе-Эйнштейна, $y_{l}=\delta_{l, 1}$ для статистики Максвелла-Больцмана и $y_{l}=1-(K+1) \delta(l /(K+1)-[l /(K+1)])$ для парастатистики. Статистическая сумма модели ядерной мультифрагментации с сохранением барионного заряда также приводится к сумме с мультиномиальными коэффициентами, в которой $y_{k \mu}=(\mp 1)^{\mu+1}$ для статистик Бозе-Эйнштейна и Ферми-Дирака, $y_{k \mu}=\delta_{\mu, 1}$ для статистики Максвелла-Больцмана и $y_{k \mu}=1-\left(K_{k}+1\right) \delta\left(\mu /\left(K_{k}+1\right)-\right.$ $\left.\left[\mu /\left(K_{k}+1\right)\right]\right)$ для парастатистики. Показано, что производяшая функция $\mathcal{Q}_{A}\left(\omega_{1}, \ldots, \omega_{A}\right)$ мультиномиальных коэффициентов $C\left(A,\left\{N_{k}\right\}\right)$ удовлетворяет рекуррентным соотношениям (16) независимо от статистики фрагментов (частиш). Сохраняющийся барионный заряд в статистической модели ядерной мультифрагментации обладает групповми свойствами [22], [23]. 
Построена точно решаемая статистическая модель ядерной мультифрагментации с учетом законов сохранения барионного и электрического зарядов. Найдена система рекуррентных уравнений для точного расчета статистической суммы со всеми четырьмя статистиками. Получены рекуррентные формулы для некоторых средних по ансамблю. Такая модель может быть использована для расчета различных характеристик, описываюших явление ядерной мультифрагментации.

\section{ПРИЛОЖЕНИЕ А}

Приведем доказательство тождества (5) для всех рассмотренных выше статистик. Пусть частицы системы подчиняются статистике Бозе-Эйнштейна, тогда правая часть тождества (5) приводится к ее левой части путем замены переменной суммирования и введением ступенчатой функции $\theta(n)$ :

$$
\begin{aligned}
& \sum_{l=1}^{N} x^{l} \sum_{n_{\vec{p}}=0}^{N-l} x^{n_{\vec{p}}} \Phi\left(N-l-n_{\vec{p}}\right)=\sum_{l=1}^{N} \sum_{n_{\vec{p}}=l}^{N} x^{n_{\vec{p}}} \Phi\left(N-n_{\vec{p}}\right)= \\
& \quad=\sum_{n_{\vec{p}}=0}^{N} \sum_{l=1}^{N} \theta\left(n_{\vec{p}}-l\right) x^{n_{\vec{p}}} \Phi\left(N-n_{\vec{p}}\right)=\sum_{n_{\vec{p}}=0}^{N} n_{\vec{p}} x^{n_{\vec{p}}} \Phi\left(N-n_{\vec{p}}\right),
\end{aligned}
$$

где $\theta(n)=1$ при $n \geqslant 0$ и $\theta(n)=0$ при $n<0$, а также $\sum_{l=1}^{N} \theta\left(n_{\vec{p}}-l\right)=n_{\vec{p}}$. Для статистики Ферми-Дирака тождество (5) выполняется в силу того, что все слагаемые ее правой части при степенях $x$ выше первой взаимно уничтожаются. Таким образом,

$$
\begin{aligned}
& \sum_{l=1}^{N}(-1)^{l+1} x^{l} \sum_{n_{\vec{p}}=0}^{\min [N-l, 1]} x^{n_{\vec{p}}} \Phi\left(N-l-n_{\vec{p}}\right)=x[\Phi(N-1)+x \Phi(N-2)]- \\
& \quad-x^{2}[\Phi(N-2)+x \Phi(N-3)]+\cdots+(-1)^{N} x^{N-1}[\Phi(1)+x \Phi(0)]+ \\
& \quad+(-1)^{N+1} x^{N} \Phi(0)=\sum_{n_{\vec{p}}=0}^{1} n_{\vec{p}} x^{n_{\vec{p}}} \Phi\left(N-n_{\vec{p}}\right) .
\end{aligned}
$$

Если частицы системы подчиняются статистике Максвелла-Больцмана, то

$$
\sum_{l=1}^{N} \delta_{l, 1} x^{l} \sum_{n_{\vec{p}}=0}^{N-l} \frac{x^{n_{\vec{p}}}}{n_{\vec{p}} !} \Phi\left(N-l-n_{\vec{p}}\right)=\sum_{n_{\vec{p}}=0}^{N} n_{\vec{p}} \frac{x^{n_{\vec{p}}}}{n_{\vec{p}} !} \Phi\left(N-n_{\vec{p}}\right) .
$$

В случае, когда частицы системы подчиняются парастатистике, тождество (5) имеет вид

$$
\begin{gathered}
\sum_{l=1}^{N}\left[1-(K+1) \delta\left(\frac{l}{K+1}-\left[\frac{l}{K+1}\right]\right)\right] x^{l} \sum_{n_{\vec{p}}=0}^{\min [N-l, K]} x^{n_{\vec{p}}} \Phi\left(N-l-n_{\vec{p}}\right)= \\
=\sum_{n_{\vec{p}}=0}^{K} n_{\vec{p}} x^{n_{\vec{p}}} \Phi\left(N-n_{\vec{p}}\right) .
\end{gathered}
$$

Легко показать, что все слагаемые левой части этого равенства при степенях $x$ выше $K$ взаимно уничтожаются. 
ПРИЛОЖЕНИЕ Б

Докажем справедливость рекуррентной формулы (15). Для этого в выражение (14) для статистической суммы $\mathcal{Q}_{A}$ в силу закона сохранения барионного $A$ заряда системы вставим единичный множитель в виде $1=\sum_{i \vec{p}} i N_{i \vec{p}} / A$,

$$
\mathcal{Q}_{A}=\frac{1}{A} \sum_{i \vec{p}^{\prime}} i \sum_{\left\{N_{k \vec{p}}\right\}} \delta\left(\sum_{k \vec{p}} k N_{k \vec{p}}-A\right) N_{i \vec{p}^{\prime}} G\left(\left\{N_{k \vec{p}}\right\}\right) e^{-\beta \sum_{k \vec{p}} E_{k \vec{p}} N_{k \vec{p}}}
$$

Заметим, что сумма по $N_{i \vec{p}^{\prime}}$ совпадает с левой частью тождества $(20)$, если положить

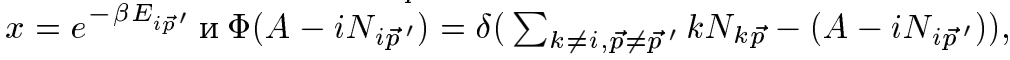

$$
\begin{aligned}
\mathcal{Q}_{A}= & \frac{1}{A} \sum_{i \vec{p}^{\prime}} i \sum_{\left\{N_{k \vec{p}}\right\}_{k \neq i, \vec{p} \neq \vec{p}^{\prime}}} G\left(\left\{N_{k \vec{p}}\right\}_{k \neq i, \vec{p} \neq \vec{p}^{\prime}}\right) e^{-\beta \sum_{k \neq i, \vec{p} \neq \vec{p}^{\prime}} E_{k \vec{p}} N_{k \vec{p}} \times} \\
& \times \sum_{N_{i \vec{p}^{\prime}}=0}^{K_{i}} N_{i \vec{p}^{\prime}} G\left(N_{i \vec{p}^{\prime}}\right) e^{-\beta E_{i \vec{p}^{\prime}} N_{i \vec{p}^{\prime}} \delta}\left(\sum_{k \neq i, \vec{p} \neq \vec{p}^{\prime}} k N_{k \vec{p}}-\left(A-i N_{i \vec{p}^{\prime}}\right)\right) .
\end{aligned}
$$

Используя тождество (20), получаем

$$
\begin{aligned}
\mathcal{Q}_{A}= & \frac{1}{A} \sum_{i \vec{p}^{\prime}} i \sum_{\left\{N_{k \vec{p}}\right\}_{k \neq i, \vec{p} \neq \vec{p}^{\prime}}} G\left(\left\{N_{k \vec{p}}\right\}_{k \neq i, \vec{p} \neq \vec{p}^{\prime}}\right) e^{-\beta \sum_{k \neq i, \vec{p} \neq \vec{p}^{\prime}} E_{k \vec{p}} N_{k \vec{p}}} \sum_{\mu=1}^{[A / i]} y_{i \mu} e^{-\beta \mu E_{i \vec{p}^{\prime}} \times} \\
& \times \sum_{N_{i \vec{p}^{\prime}}=0}^{\min \left([A / i]-\mu, K_{i}\right)} G\left(N_{i \vec{p}^{\prime}}\right) e^{-\beta E_{i \vec{p}^{\prime}} N_{i \vec{p}^{\prime}} \delta}\left(\sum_{k \neq i, \vec{p} \neq \vec{p}^{\prime}} k N_{k \vec{p}}-\left(A-i N_{i \vec{p}^{\prime}}-i \mu\right)\right) .
\end{aligned}
$$

Переставляя суммы по числам заполнения и переменной суммирования $\mu$, а также используя определение (14), окончательно получаем требуемое рекуррентное уравнение $(15)$,

$$
\begin{aligned}
\mathcal{Q}_{A}= & \frac{1}{A} \sum_{i} i \sum_{\mu=1}^{[A / i]} y_{i \mu} \sum_{\vec{p}^{\prime}} e^{-\beta \mu E_{i \vec{p}^{\prime}} \times} \\
& \times \sum_{\left\{N_{k \vec{p}}\right\}} \delta\left(\sum_{k \vec{p}} N_{k \vec{p}}-(A-i \mu)\right) G\left(\left\{N_{k \vec{p}}\right\}\right) e^{-\beta \sum_{k \vec{p}} E_{k \vec{p}} N_{k \vec{p}}}= \\
= & \frac{1}{A} \sum_{i} \sum_{\mu=1}^{[A / i]} i \omega_{i \mu} \mathcal{Q}_{A-i \mu} .
\end{aligned}
$$

Благодарности. Автор благодарит В. Д. Тонеева, М. Плосжайчика и К. К. Гудиму за исключительно полезные обсуждения, а также В. В. Ужинского и Ю. Б. Иванова за важные замечания. 


\section{Список литературы}

[1] J.P. Bondorf, A.S. Botvina, A.S. Iljinov, I. N. Mishustin, K. Sneppen. Phys. Rep. 1995. V. 257. P. 133.

[2] D. H. E. Gross. Rep. Prog. Phys. 1990. V. 53. P. 605.

[3] R. Hagedorn, K. Redlich. Z. Phys. C. 1985. V. 27. P. 541.

[4] J. Cleymans, H. Oeshler, K. Redlich. Phys. Rev. C. 1999. V. 59. P. 1663.

[5] Джс. Риордан. Введение в комбинаторный анализ. М.: ИЛ, 1963.

[6] P. T. Landsberg. Thermodynamics. New York: Interscience, 1961.

[7] P. Borrmann, G. Franke. J. Chem. Phys. 1993. V. 98. P. 2484.

[8] H.-J. Schmidt, J. Schnack. Physica A. 1998. V. 260. P. 479.

[9] A.S. Parvan, V. D. Toneev, M. Ptoszajczak. Nucl. Phys. A. 2000. V. 676. P. 409.

[10] K. C. Chase, A.Z. Mekjian. Phys. Rev. C. 1995. V. 52. P. R2339.

[11] S. Das Gupta, A.Z. Mekjian. Phys. Rev. C. 1998. V. 57. P. 1361.

[12] K. C. Chase, A. Z. Mekjian. Phys. Rev. C. 1994. V. 50. P. 2078.

[13] S. Pratt, S. Das Gupta. Phys. Rev. C. 2000. V. 62. P. 044603.

[14] А. С. Парван, В. Д. Тонеев, К. К. Гудима. ЯФ. 1999. Т. 62. С. 1593.

[15] P. Bhattacharyya, S. Das Gupta, A.Z. Mekjian. Phys. Rev. C. 1999. V. 60. P. 054616; P. 064625 .

[16] К. Хуанг. Статистическая механика. М.: Наука, 1966.

[17] И. А. Квасников. Термодинамика и статистическая физика. Теория равновесных систем. М.: Изд-во МГУ, 1991.

[18] М. Хамермеш. Теория групп и ее применения к физическим проблемам. М.: Мир, 1966.

[19] A. Z. Mekjian, S. J. Lee. Phys. Rev. A. 1991. V. 44. P. 6294.

[20] A. B. Balantekin. Phys. Rev. E. 2001. V. 64. P. 066105.

[21] H.-J. Schmidt, J. Schnack. Am. J. Phys. 2002. V. 70. P. 53.

[22] F. Cerulus. Nuovo Cimento. 1961. V. 19. P. 528.

[23] K. Redlich, L. Turko. Z. Phys. C. 1980. V. 5. P. 201.

[24] А. Парван. Ядерная мультифрагментация в статистическом подходе. Дисс. канд. физ.-мат. наук. Дубна: ОИЯИ, 2002.

Поступила в редакцию 12. III.2003 г., после доработки 9.VII.2003 г. 